\title{
Communication \\ Phase Measurement of Guided-Mode Resonance Device Using Digital Micromirror Device Gratings
}

\author{
Min-Xu Chiang, Jaturon Tongpakpanang and Wen-Kai Kuo * \\ Graduate Institute of Electro-Optical and Materials Science, National Formosa University, Yunlin 63208, Taiwan; \\ MinXu.Chiang@auo.com (M.-X.C.); d0677104@gm.nfu.edu.tw (J.T.) \\ * Correspondence: wkkuo@nfu.edu.tw
}

\begin{abstract}
This paper reports on the measurement system of the phase difference between s- and $p$-polarization components of the light passing through a guided-mode resonance (GMR) device using a digital micromirror device (DMD) gratings as a digital phase-shifting device. The phase of the non-zeroth order diffraction beams of the grating pattern displayed on the DMD can exhibit a phase change when the grating pattern is shifted. Two nearest different diffraction orders of $p$-polarized and s-polarized beams can be used as the reference and measurement beams, respectively, and are combined to implement the phase-shifting interferometry (PSI). The phase difference between the $s$ - and the $p$-polarization components of the incident light passing through the GMR device can be obtained by applying the four-step phase-shift algorithm to the DMD-based PSI system. Experimental results show that this measurement system has a phase detection limit of $1^{\circ}$ and was able to obtain the abrupt phase difference curve of the GMR device versus the incident angle.
\end{abstract}

Keywords: guided-mode resonance; digital micromirror; phase-shifting interferometry

check for

updates

Citation: Chiang, M.-X.;

Tongpakpanang, J.; Kuo, W.-K. Phase

Measurement of Guided-Mode

Resonance Device Using Digital

Micromirror Device Gratings.

Photonics 2021, 8, 136. https://

doi.org/10.3390/photonics 8050136

Received: 29 March 2021

Accepted: 22 April 2021

Published: 23 April 2021

Publisher's Note: MDPI stays neutral with regard to jurisdictional claims in published maps and institutional affiliations.

Copyright: (c) 2021 by the authors. Licensee MDPI, Basel, Switzerland. This article is an open access article distributed under the terms and conditions of the Creative Commons Attribution (CC BY) license (https:/ / creativecommons.org/licenses/by/ $4.0 /)$.

\section{Introduction}

A guided-mode resonance (GMR) device consists of a waveguide layer and grating structure, and incident light can be coupled to the waveguide layer by the grating high diffraction orders. Because of the grating, the propagation wave inside the waveguide layer slowly leaks out of the waveguide guide, and when the incident light wavelength is at the resonance wavelength, the leakage wave interferes with the non-diffraction wave to produce nearly $0 \%$ transmittance and $100 \%$ reflectivity as a filter response [1]. This resonance angle is highly sensitive to the refractive index (RI) of material on the grating surface; therefore, the GMR device can be used as a label-free biosensor [2]. Among the GMR sensor interrogation methods, the phase-based interferometric approach has been proved to have a better detection resolution [3,4]. In our previous works, heterodyne interferometry based on an electro-optic modulator was presented [5] and subsequently, phase-shift interferometry (PSI) based on two liquid-crystal (LC) retardation plates was also proposed [6]. Our results showed that the phase-detection sensitivity of the RI change on the grating surface of the GMR device can be better than $5000^{\circ} /$ RIU. Since the PSI is an image-type detection, it can achieve parallel (multi-channel) sensing and has great potential for high-throughput screening. In this paper, we propose using a digital micromirror device (DMD) to replace the LC device in our previous work to implement the PSI system. The LC device in the PSI needs a calibration process to obtain an accurate analog driving voltage. The DMD-based spatial light modulator (SLM) can display shifting grating patterns to achieve four digital phase-shifting, $0, \pi / 2, \pi$, and $3 \pi / 2$, without the calibration process. A PSI system implemented by an electrically addressed liquid-crystal-based SLM has been proposed [7]. Here, the DMD-SLM is proposed to substitute the LC-SLM because the DMDSLM has a better reflectivity and higher operation speed $[8,9]$. We propose to use the DMDSLM to facilitate digital phase-shifting in the PSI system using proper micro-mirror patterns without the need for the accurate analog driving voltage in the LC device. We also propose 
a method to use this DMD-SLM-based PSI system to perform the phase measurement of the GMR device. Besides, because of mature batch-type production technologies, DMD chips have become cost-effective and more attractive for many applications. The diffraction properties of the DMD-SLM are different from the LC-SLM. We will first investigate diffraction patterns of the DMD with different grating patterns and then describe how to utilize the DMD-SLM in the PSI system. Subsequently, fabrication and simulation of the GMR device will be briefly reviewed. Finally, we will demonstrate the measurement results of the phase difference between the $s$ - and p-polarization of the GMR sensor using this DMD-based PSI system.

\section{DMD Grating and Its Diffraction Pattern}

A schematic of the setup for observation of the diffraction patterns of a DMD grating is illustrated in Figure 1. A He-Ne laser with a wavelength of $632.8 \mathrm{~nm}$ was normally incident to the DMD surface through a hole in a black-color paper plate that was $1 \mathrm{~m}$ away from the DMD surface, and light spots of the reflective diffraction beams could be observed on the paper plate. Because the DMD was a two-dimensional (2D) grating pattern, diffraction spots along the vertical and horizontal directions were expected to be observed. The schematic of all the DMD pixels in the static state (without applying voltage) is as shown in Figure 2a, in which all micromirrors were parallel to the substrate. The pitch (period) of the 2D gratings is equal to the width of a pixel cell as indicated in Figure 2a [10]. Its diffraction spot image is shown in Figure 2b. Multiple order diffraction light spots were observed, and the zeroth-order light reflected through the hole in the paper plate. The DMD 2D array pixels can be mathematically treated as a convolution of the rectangle ("rect") and the comb ("comb") functions. Let the Fourier transforms of the "rect" and the "comb" functions be "Sinc" and "Comb" functions, respectively. Therefore, the diffraction patterns of the DMD can be calculated as the multiplication of the "Sinc" and "Comb" functions [11]. In Figure 2b, the center (peak intensity) of the 2D "Sinc" envelope function is the zero-other reflected spot position (hole), and the "Comb" spots near the hole have a higher intensity. The location of the "Sinc" envelope peak is dependent on the incident angle and tilted angle of the micromirror [12]. According to the grating equation, spacing $D$ between two nearest diffraction order spots is inversely proportional to the grating period.

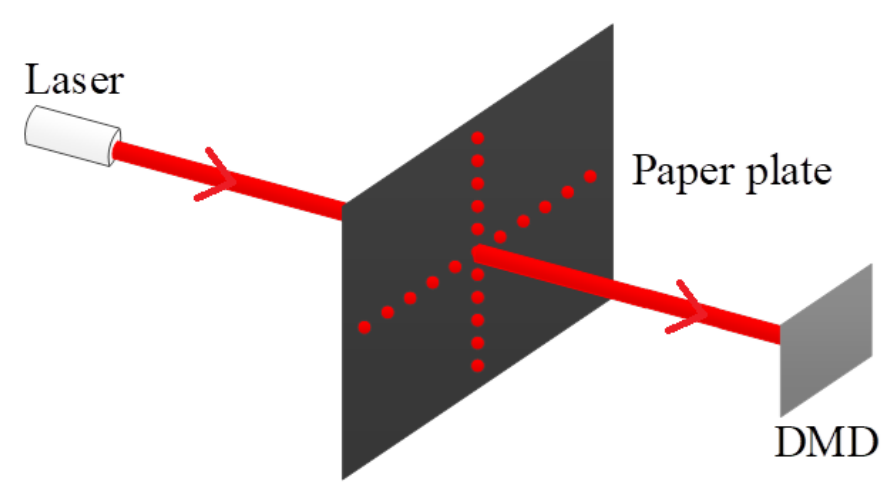

Figure 1. Schematic of setup for observation of the diffraction patterns of a DMD.

When proper voltages are applied, the micromirror can be tilted to $+12^{\circ}$ or $-12^{\circ}$ along its diagonal, corresponding to the ON- or OFF-state, respectively. The schematic of all the micromirrors in the ON-state is shown in Figure 2c. The grating structure becomes the brazed-type, and its period is indicated as $\Lambda_{1}$; its corresponding diffraction spot image is shown in Figure 2d. The tilted micromirrors cause the center peak of the "Sinc" envelope to shift from the hole to a new location along the $45^{\circ}$ line. In this figure, the hole in the paper plate is labeled $O$ as the reference point. When all the micromirrors are in the OFF-state, the schematic and its diffraction spot image are as shown in Figure 2e,f, respectively. The center peak of the "Sinc" envelope is shifted to the opposite side. 


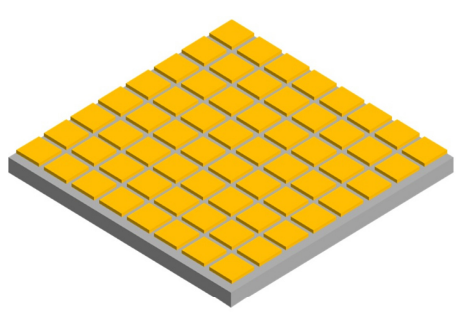

(a)

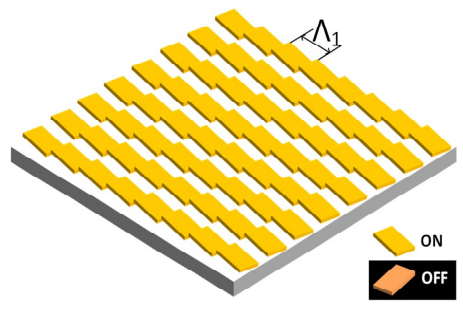

(c)

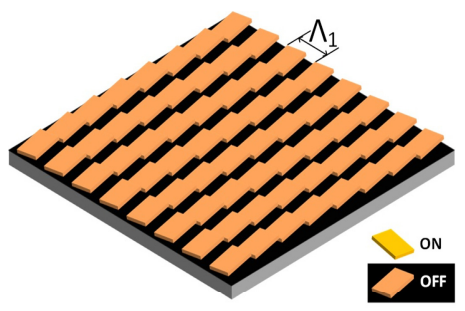

(e)

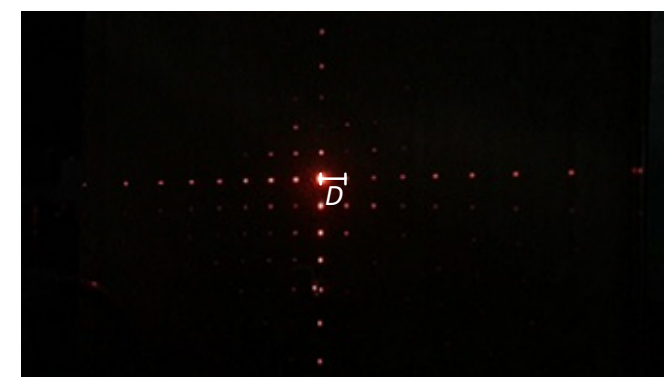

(b)

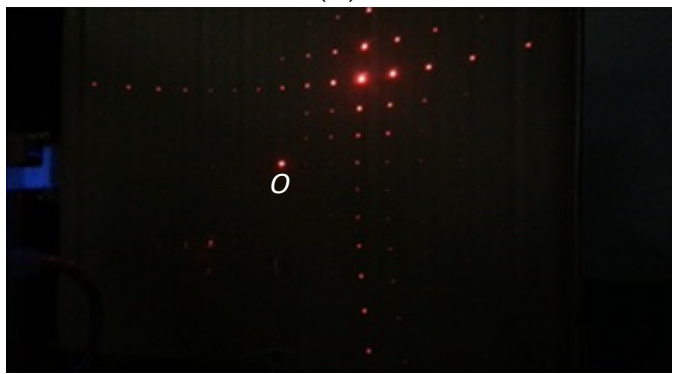

(d)

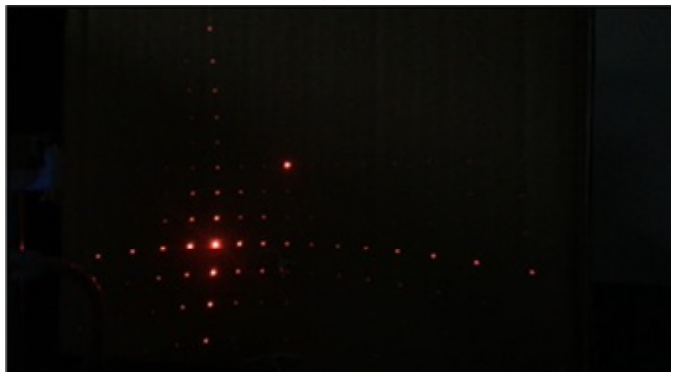

(f)

Figure 2. (a) Schematic of all micromirror pixels in the static state, and (b) its diffraction spot image. (c) Schematic of all micromirror pixels in $\mathrm{ON}$-state (tilted $+12^{\circ}$ ), and (d) its diffraction spot image. (e) Schematic of all micro-mirror pixels in OFF-state (tilted $-12^{\circ}$ ), and (f) its diffraction spot image.

Next, two modulated DMD patterns were investigated. Figure 3a shows the schematic of a DMD pattern with a period of one line ON-state (white stripe) and one line OFF-state (black stripe). In this configuration, the grating period indicated as $\Lambda_{2}$ was two DMD pixel widths. Because of the two opposite-tilted angles $\left(+12^{\circ}\right.$ and $\left.-12^{\circ}\right)$ of the micromirrors, two symmetric diffraction spot images were observed on two sides of the center along the $-45^{\circ}$ line, as shown in Figure $3 b$. Because the line grating period $\Lambda_{2}$ was double that of $\Lambda_{1}$ in Figure $2 c$, the distance between the two nearest order diffraction spots along the horizontal direction became half of that in Figure 2c. Because there was no pattern modulation along the other vertical direction, the distance between the two nearest diffractive spots along the vertical direction was the same as that in Figure 2c. To implement the digital four-phase shifting, a DMD pattern with a period of $\Lambda_{3}$ having four-pixel width, including two ONstate (white stripe) and two OFF-state (black stripe) line pixels, was required. Its schematic and corresponding diffraction spot image are shown in Figure $3 c, d$, respectively. Because the grating pitch $\Lambda_{3}$ was twice $\Lambda_{2}$, the distance between two diffraction spots along the horizontal direction became only half of that in Figure $3 \mathrm{~b}$. The phase difference between the two nearest order diffraction lights could be changed by shifting this DMD line pattern to implement the phase shifting in a PSI system. The number of reflecting mirrors and their periods did not affect the performance of the PSI system. The mirror period will change the diffraction angle only. 


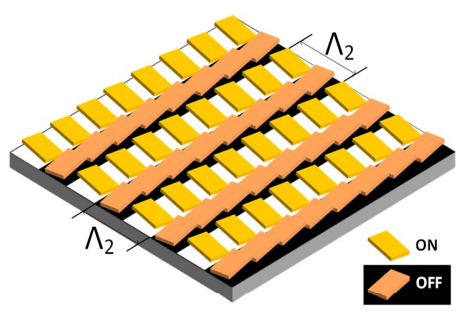

(a)

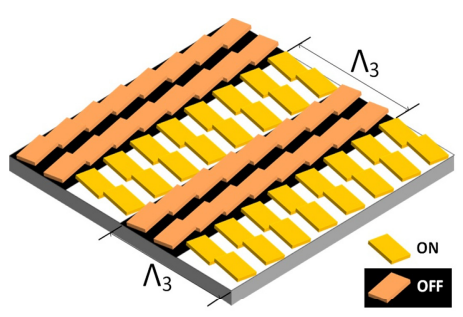

(c)

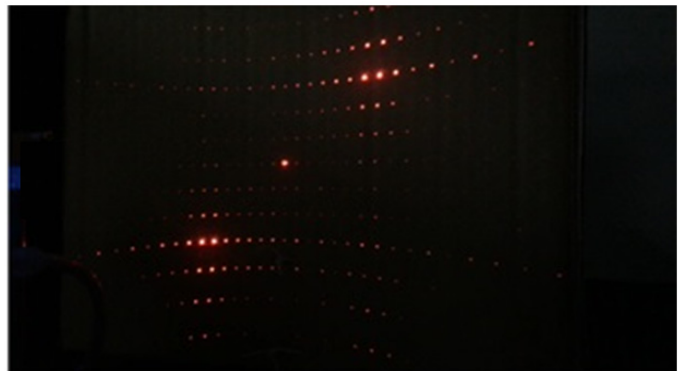

(b)

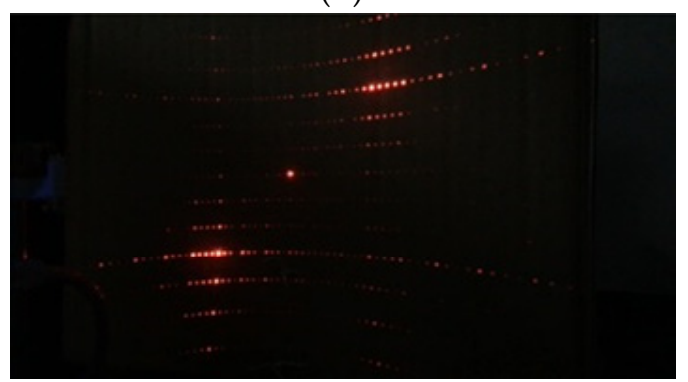

(d)

Figure 3. (a) Schematic of the DMD pattern with a period of $1 \mathrm{ON}$-state and 1 OFF-state line pixel, and (b) its diffraction spot pattern. (c) Schematic of DMD grating pattern with a period of $2 \mathrm{ON}$-state and 2 OFF-state line pixels, and (d) its diffraction pattern.

\section{DMD-Based PSI System}

Figure 4 shows the optical setup of the proposed DMD-based PSI system. Here, we used a commercially available DLP LightCrafter display 2000 evaluation module (DLPDLCR 2000EVM, Texas Instruments), comprising a DLP 2000 DMD chip with a resolution of $640 \times 480$. This is a development kit for micro-projectors and can be controlled via a Beaglebone Black platform. In our system, a He-Ne laser was used as the light source, and two polarizers, $\mathrm{P}_{0}$ and $\mathrm{P}_{1}$, were used to vary the incident light intensity. $\mathrm{P}_{1}$ was rotated $45^{\circ}$ to yield output light having both $p$ - and s-polarized components. The output light passed through a Wollaston prism (WP) and split into an s-polarized beam (red line) and a $p$-polarized beam (green line) at a separation angle of $15^{\circ}$. The $p$-polarized beam was directly incident on the DMD chip, while the s-polarized beam passed two mirrors $\left(\mathrm{M}_{2}\right.$ and $\mathrm{M}_{3}$ ) before being incident on the DMD chip. These two mirrors were used to adjust the zeroth-order reflected beam (solid red line) of the s-polarized light to coincide with the first-order reflected beam (dashed green line) of the $p$-polarized incident light. The angle difference $\theta$ between these two incident beams was equal to the difference between the zeroth- and the first-order reflected beams of the $p$-polarized light. The phase difference between these two coincided beams could be controlled by the four-line pattern displayed on the DMD chip. This coincided beam was then passed through an aperture (AP) and analyzer (A) and became a measurement beam in the PSI system. After this beam passed through the GMR device, the resulting interferograms were generated and captured by a web camera (WebCAM) (Phillips 900nc). A lens (L) was used to adjust the image size of the interferograms. Diffraction spot patterns of these two reflected beams before and after coinciding are shown in Figure 5a,b, respectively. The intensity difference between the sand $p$-polarized beams could be adjusted by rotating the analyzer to have the best contrast of the interferogram. The final enlarged interferogram is shown in Figure $5 c$. 


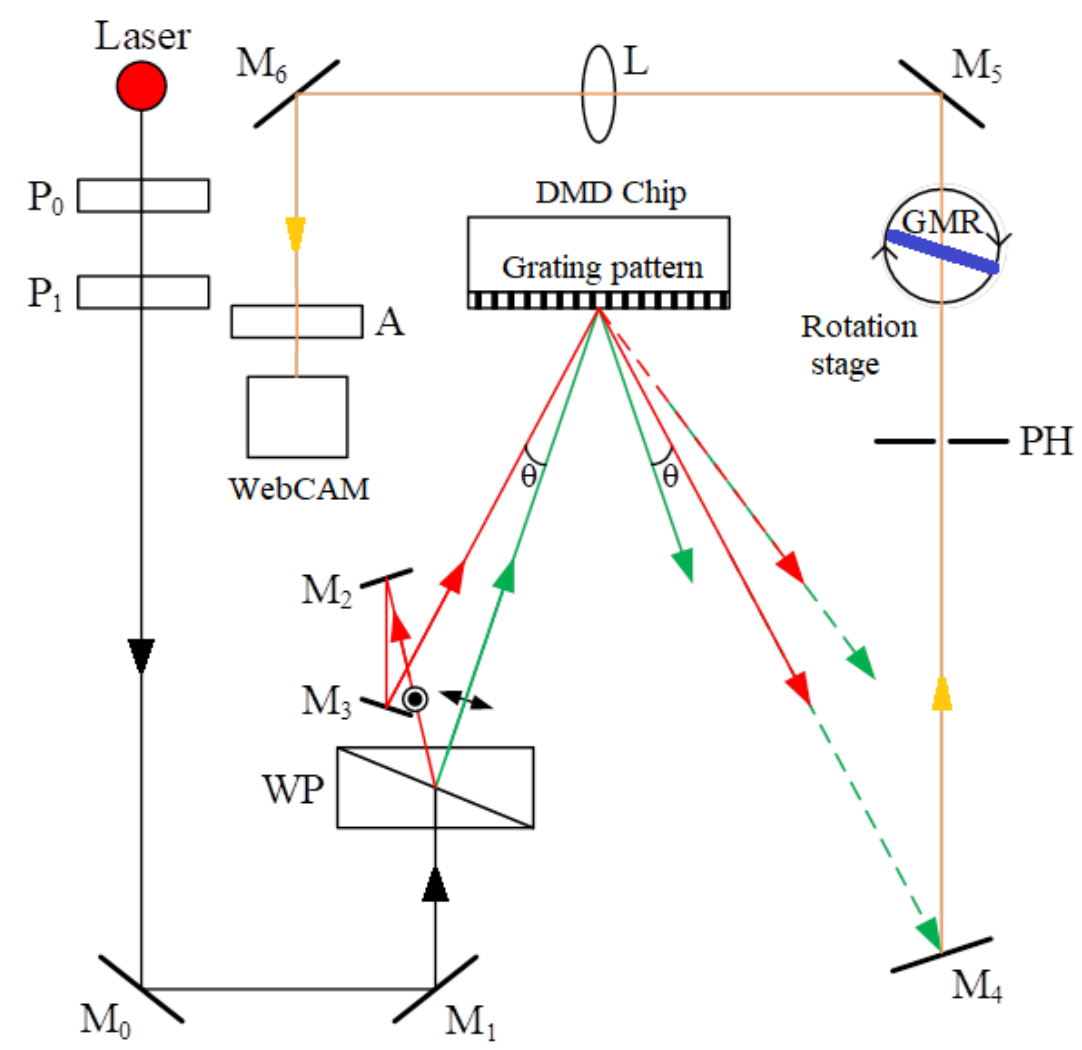

Figure 4. The optical setup of the proposed digital PSI based on the DMD.

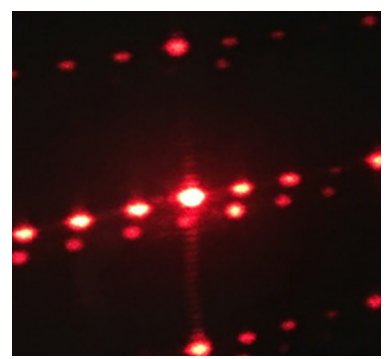

(a)

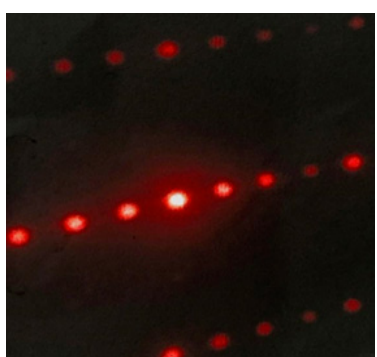

(b)

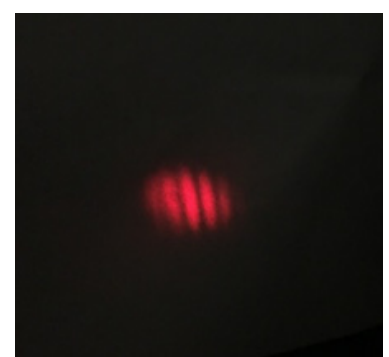

(c)

Figure 5. Diffraction spot patterns of the $s$ - and the $p$-polarized reflected beams (a) before and (b) after coinciding alignment. (c) The final enlarged interferogram.

A set of four-step patterns displayed on the DMD-SLM is shown in Figure 6. The black- and white-stripe colors correspond to two lines of OFF- and ON-states, respectively, on the DMD chip as illustrated in Figure 3c. Each subsequent pattern had one pixel shifting to achieve a phase shift of $\pi / 2$. Therefore, four digital phase shifts, $0, \pi / 2, \pi$, and $3 \pi / 2$, could be achieved by sequentially displaying these four patterns on the DMD-SLM. Before performing the GMR sensor measurements, system repeatability was investigated. The GMR device was replaced by a null glass substrate and four interferograms corresponding to the four patterns of Figure 6 were obtained, as shown in Figure 7. Because the action of the micromirrors was flipped, not stationary, this could have a time-averaging effect on the laser intensity and reduce the speckle in the interferogram. In each interferogram image, a red square area $(10 \times 10$ pixels $)$ was selected, as indicated in the figure, and their average gray values were used to obtain the average intensity values used in the PSI system. The phase difference between the $s$ - and $p$-polarized lights could be calculated with the 4-step phase-shifting algorithm [13]. The same condition was repeatedly measured 8 times, and hence, eight phase values were calculated; the results are shown in Figure 8. The upper 
curve (black) represents the 32 average intensity gray values of the eight sets, whereas the red curve (red) represents the calculated phase values. The variation of the phase values was within $1^{\circ}$ and hence, the system detection limit could be estimated to be $1^{\circ}$. This detection limit may be further improved by enhancing the stiffness of the PSI system.

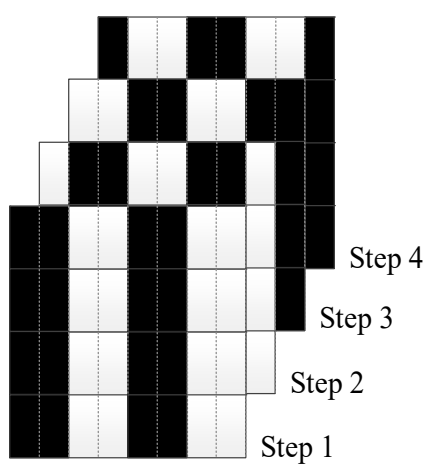

Figure 6. Four-step patterns displayed on the DMD-SLM. Each subsequent step pattern with one pixel shifting corresponding to a phase shift of $\pi / 2$ in the first order diffraction beam.

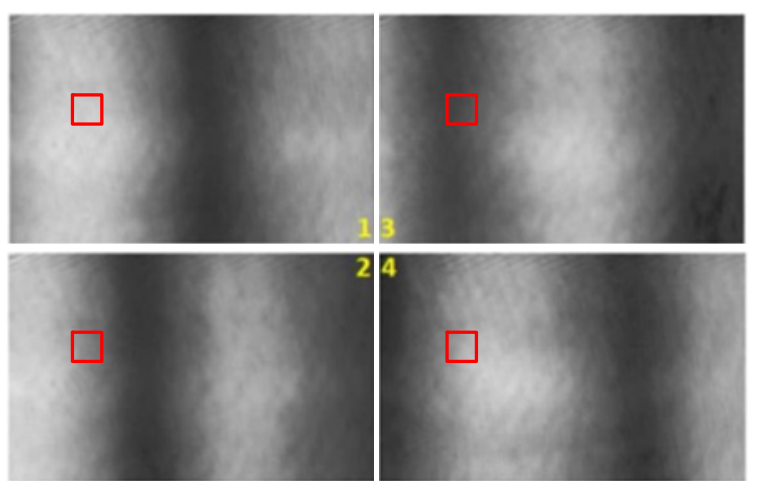

Figure 7. Four-step interferograms (1 4) of the null glass substrate corresponding to the four-step patterns in Figure 6 . A selected square area $(10 \times 10$ pixels, in a red-color line $)$ for calculating PSI in the same position for each interferogram.

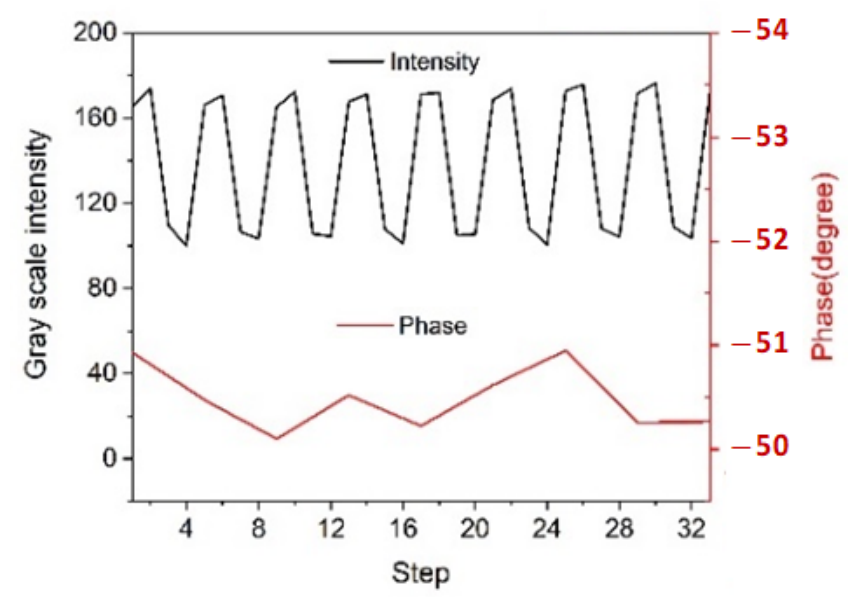

Figure 8. Stability test results for the experimental setup.

\section{GMR Device Measurement Results}

Finally, the setup was used to measure the phase difference between the $s$ - and $p$ polarized light beams passing through the GMR device. Under the resonance condition, the angular transmission phase of the GMR device had a large phase change known as 
Goos-Hänchen (GH) shift [14,15]. The resonance angles of the GMR device for the s- and p-polarized light beams were different, and the proposed DMD-based PSI system was able to measure the phase difference between the resonance angle and non-resonance angle. The schematic of the tested GMR device is shown in Figure 9a; the device is similar to the one in our previous report [6]. The device was fabricated on a glass substrate and comprised a $\mathrm{TiO}_{2}$ thin-film layer with a thickness $d$ of $325 \mathrm{~nm}$, a SiO 2 grating layer with a period $\Lambda$ of $555 \mathrm{~nm}$, and modulation depth $h$ of $65 \mathrm{~nm}$. The grating structure was fabricated by using the nanoimprinting sol-gel method. A photograph of the completed GMR sensor is shown in Figure 9b.

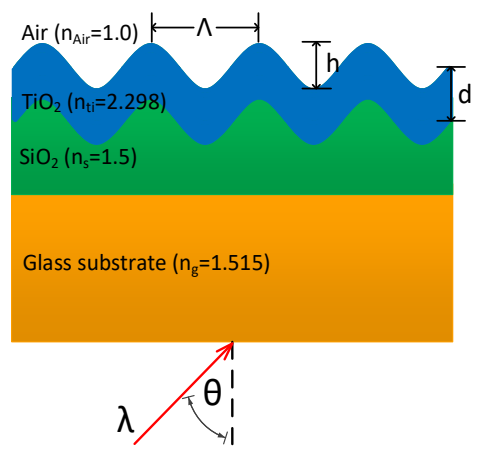

(a)

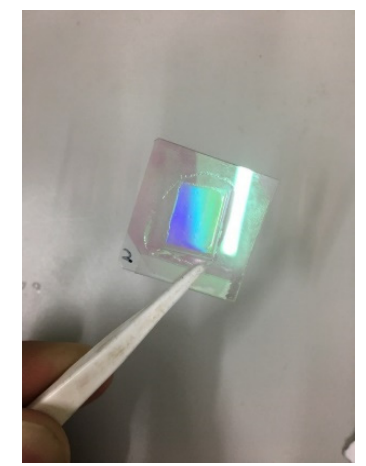

(b)

Figure 9. (a) Schematic and (b) photo of the GMR sensor.

The GMR device was simulated using commercially available finite-difference timedomain (FDTD) software. The GMR device was investigated with the condition that the $p$-polarized component of the incident light satisfy the phase-matching condition. The incident light entered at the substrate of the GMR device and the sensor surface was air $(n=1.0)$. In simulation, complex refractive $n=2.298$ and extinction coefficient $k=0.005$ of the $\mathrm{TiO}_{2}$ thin-film layer were used. The extinction coefficient was used to model the loss due to all non-ideal effects. The simulation results for the transmissivity versus the scanning incident angle are shown in Figure 10a. The resonance $p$-polarization and the non-resonance s-polarization are indicated as the red and black lines, respectively, and the resonance angle of $p$-polarization is $18.8^{\circ}$. The transmission phases of the GMR device are shown in Figure 10b. The resonance transmission phase ( $p$-polarization, red) had an abrupt change near the resonance angle, while the non-resonance phase (s-polarization, black) had a smooth change due to the incident angle change. The phase difference between the $p$ and the s-polarization component is also shown in Figure 10b. This phase difference can be measured by the proposed DMD-based PSI system.

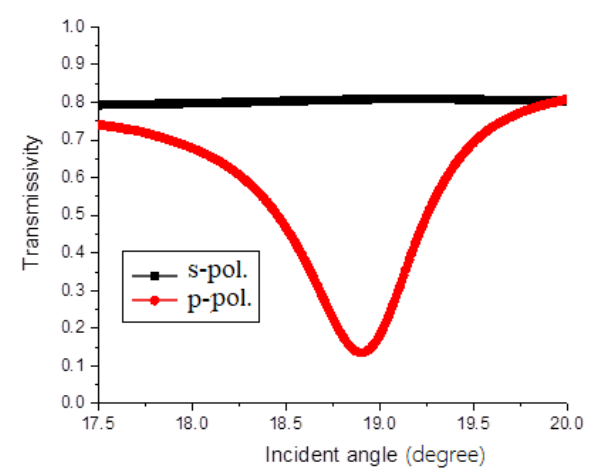

(a)

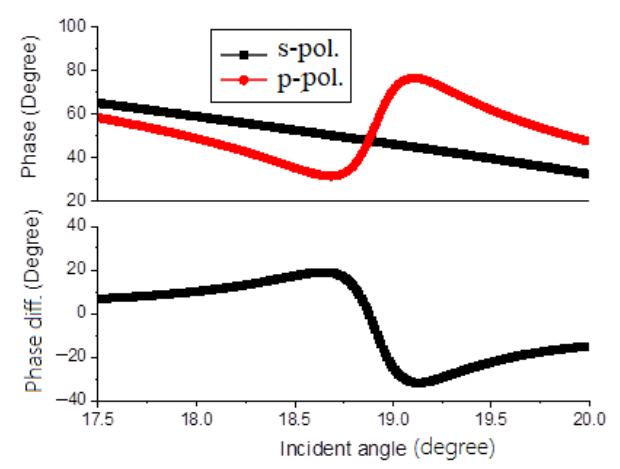

(b)

Figure 10. Simulation results for the (a) transmittance, (b) phase curves, and phase difference between, the polarization components of the incident light. 
For the measurements, the GMR device was mounted on a rotation stage as indicated in Figure 4 , and the incident angle was varied from $16^{\circ}$ to $18.5^{\circ}$ with an interval of $0.05^{\circ}$ by rotating the stage. Five selected sets of four-step interferograms $\left(\mathrm{I}_{1} \sim \mathrm{I}_{4}\right)$ (in a red-color rectangle line) corresponding to four incident angles, $17^{\circ}, 17.25^{\circ}, 17.5^{\circ}, 17.75^{\circ}$, and $18^{\circ}$, are shown in Figure 11, and each set of the four-step interferograms was used to calculate phase values by applying the 4-step phase-shifting algorithm to the selected square area, as shown in Figure 7. The five sets of interferograms were selected to demonstrate the variation of the grayscale values due to the resonance angle. The average intensities of the interferograms decreased from $17^{\circ}$ to $17.25^{\circ}$ as the incident angle was approaching the resonance angle, reached the minimum at the resonance angle of $17.5^{\circ}$, and then increased from $17.75^{\circ}$ to $18^{\circ}$ as the incident angle was leaving the resonance angle.

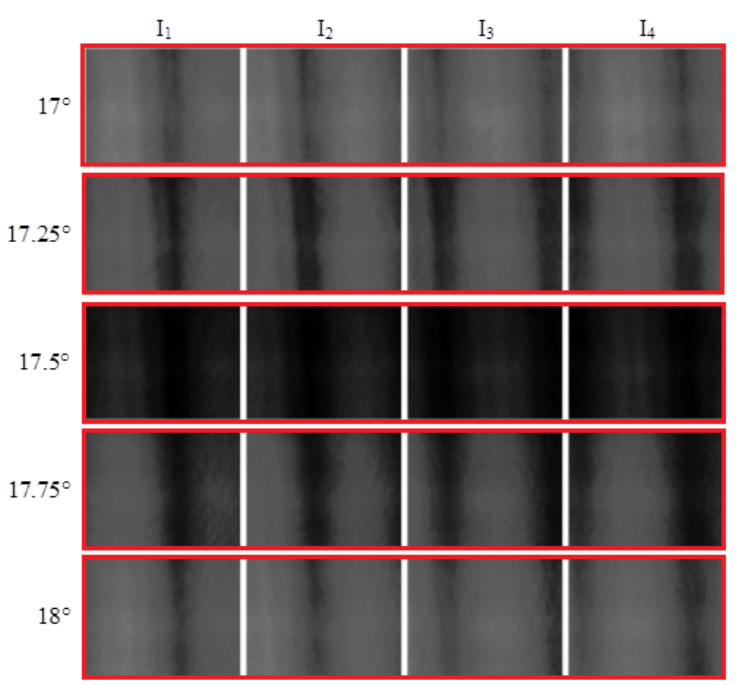

Figure 11. Five selected sets of 4 -step interferograms $\left(\mathrm{I}_{1} \sim \mathrm{I}_{4}\right)$ (in a red-color rectangle line) corresponding to 5 incident angles, $17^{\circ}, 17.25^{\circ}, 17.5^{\circ}, 17.75^{\circ}$, and $18^{\circ}$. Average intensities of the interferograms decreased from $17^{\circ}$ to $17.25^{\circ}$, reached a minimum at the resonance angle of $17.5^{\circ}$, and then increased from $17.75^{\circ}$ to $18^{\circ}$.

The calculated 50 phase values $($ red) and $200(50 \times 4)$ intensities (gray) of the selected areas in all interferograms versus the incident angle are shown in Figure 12. The obtained phase curve corresponded to the phase difference between $s$ - and $p$-polarization components and agreed with the simulation in Figure 10b. The black line is the average transmitted intensity and corresponds to the transmissivity in Figure 10a. The resonance angle can be found as $17.5^{\circ}$, and the phase curve exhibited an abrupt change near the resonance angle. The simulation results have a larger resonance angle and phase dynamic range than the measured values; these discrepancies may be due to the parameters of the fabricated GMR device, which were not the same as those used in the simulation model. In Table 1, this system is compared with other phase measurement studies of the GMR device.

Table 1. Comparison of some studies on phase measurement of the GMR device.

\begin{tabular}{cccccc}
\hline Features & This Work & Ref. [3] & Ref. [4] & Ref. [5] & Ref. [6] \\
\hline Cost & Low & High & Fair & High & Fair \\
\hline Multi-Channel & Yes & Yes & Yes & No & Yes \\
\hline Digital-PSI & Yes & No & No & No & No \\
\hline Noise & $1^{\circ}$ & $0.3^{\circ}$ & $0.3^{\circ}$ & $0.1^{\circ}$ & $10^{\circ}$ \\
\hline
\end{tabular}




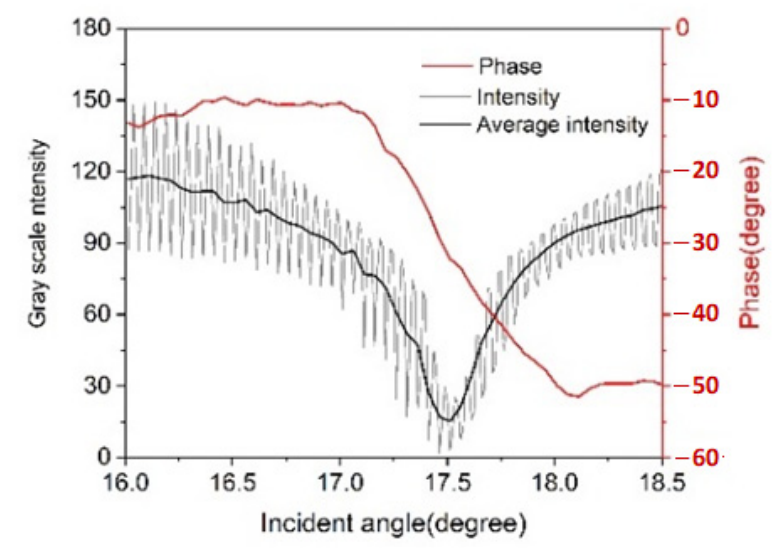

Figure 12. The total calculated phase values and successive intensities in the selected area of the recorded interferograms versus the incident angle.

\section{Conclusions}

A PSI system using the DMD-SLM as a phase shifter was developed and applied to measure the phase difference of a GMR device. The DMD grating pattern can be designed and used to digitally shift the phase of a first-order diffraction beam by one pixel width in the displayed grating pattern without calibrating the driving voltage as needed in an LC phase retarder. The experimental setup could achieve phase difference measurements with a repeatability of $1^{\circ}$, and this value may be further improved by elevating the stability of the optical system. The proposed system successfully yielded the transmitted abrupt phase curve of the GMR device and could be applied to several phase difference measurements.

Author Contributions: Conceptualization, W.-K.K.; methodology, M.-X.C.; writing-original draft preparation, J.T.; writing-review and editing, W.-K.K.; visualization, J.T.; supervision, W.-K.K.; project administration, W.-K.K.; funding acquisition, W.-K.K. All authors have read and agreed to the published version of the manuscript.

Funding: This research was funded by the Ministry of Science and Technology, Taiwan, grant number 107-2221-E-150-023, 108-2221-E-150-026, and 109-2221-E-150-07.

Institutional Review Board Statement: Not applicable.

Informed Consent Statement: Not applicable.

Data Availability Statement: Not applicable.

Acknowledgments: The authors thank Everwide Chemical Co., Ltd. of Taiwan for the free UV-cured glue that was used to fabricate GMR devices.

Conflicts of Interest: The authors declare no conflict of interest.

\section{References}

1. Wang, S.S.; Magnusson, R. Theory and applications of guided-mode resonance filter. Appl. Opt. 1993, 32, 2606-2613. [CrossRef] [PubMed]

2. Kim, W.J.; Kim, B.K.; Kim, A.; Huh, C.; Ah, C.S.; Kim, K.H.; Hong, J.; Park, S.H.; Song, S.; Song, J.; et al. Response to cardiac markers in human serum analyzed by guided-mode resonance biosensor. Anal. Chem. 2010, 82, 9686-9693. [CrossRef] [PubMed]

3. Sahoo, P.K.; Sarkar, S.; Joseph, J. High sensitivity guided-mode-resonance optical sensor employing phase detection. Sci. Rep. 2017, 7, 7607. [CrossRef] [PubMed]

4. Barth, I.; Conteduca, D.; Reardon, C.; Krauss, T.F. Common-path interferometric label-free protein sensing with resonant dielectric nanostructures. Light Sci. Appl. 2020, 9, 96. [CrossRef] [PubMed]

5. Kuo, W.K.; Huang, N.C.; Weng, H.P.; Yu, H.H. Tunable phase detection sensitivity of transmitted-type guided-mode resonance sensor in a heterodyne interferometer. Opt. Express. 2014, 22, 22968-22973. [CrossRef]

6. Kuo, W.K.; Syu, S.H.; Lin, P.Z.; Yu, H.H. Tunable sensitivity phase detection of transmitted-type dual-channel guided-mode resonance sensor based on phase-shift interferometry. Appl. Opt. 2016, 55, 903-907. [CrossRef] [PubMed] 
7. Bitou, Y. Digital phase-shifting interferometer with an electrically addressed liquid crystal spatial light modulator. Opt. Lett. 2003, 28, 1576-1578. [CrossRef]

8. Hoffmann, M.; Papadopoulos, L.N.; Judkewitz, B. Kilohertz binary phase modulator for pulsed laser sources using a digital micromirror device. Opt. Lett. 2018, 43, 22-25. [CrossRef] [PubMed]

9. Chandrasekaran, S.N.; Ligtenberg, H.; Steenbergen, W.; Vellekoop, I.M. Using digital micromirror devices for focusing light through turbid media. In Proceedings of the Emerging Digital Micromirror Device Based Systems and Applications VI 897905 (SPIE MOEMS-MEMS), San Francisco, CA, USA, 27 January-1 February 2014.

10. Son, J.Y.; Lee, B.R.; Chernyshov, O.O.; Moon, K.A.; Lee, H. Holographic display based on a spatial DMD array. Opt. Lett. 2013, 38, 3173-3176. [CrossRef] [PubMed]

11. Liu, J.P.; Horimai, H.; Lin, X.; Liu, J.Y.; Huang, Y.; Tan, X.D. Optimal micro-mirror tilt angle and sync mark design for digital micro-mirror device based collinear holographic data storage system. Appl. Opt. 2017, 56, 4779-4784. [CrossRef] [PubMed]

12. Using Lasers with DLP DMD Technology; TI DN 2509927; Texas Instruments: Dallas, TX, USA, 2008.

13. Hariharan, P.; Oreb, B.F.; Eiju, T. Digital phase-shifting interferometry: A simple error-compensating phase calculation algorithm. Appl. Opt. 1987, 26, 2504-2506. [CrossRef] [PubMed]

14. Luder, H.; Paulsen, M.; Gerken, M. Photonic crystal slab between orthogonal polarizers: Details on the guided mode resonance wavelength. Opt. Quant. Electron. 2020, 52, 180. [CrossRef]

15. Yang, R.; Zhu, W.; Li, J. Giant positive and negative Goos-Hänchen shift on dielectric gratings caused by guided-mode resonance. Opt. Express. 2014, 22, 2043-2050. [CrossRef] [PubMed] 